\title{
Liquid-Phase Electron Spectroscopy of Individual Plasmonic Nanostructures
}

\author{
Alan Dai, Michal Vadai, Katherine Sytwu and Jennifer Dionne
}

Stanford University, Stanford, California, United States

Sustainable and industrial photochemical and photocatalytic reactions, including artificial photosynthesis, organic synthesis, and pollutant degradation, require nanoparticle catalysts in liquid environments for physically significant reactivity. Plasmonic nanostructures have emerged as materials which promise enhanced efficiency and selectivity at milder conditions compared to traditional metal catalysts. [1,2] Insitu transmission electron microscopy (TEM) is one key approach towards developing more efficient plasmon catalysts, able to image and characterize these materials at the single and sub-particle level and monitor their properties at finely resolved spatial and temporal coordinates throughout a reaction. While considerable advances have been made in monitoring plasmon properties and plasmon-driven reactions with sub-nanometer spatial and millisecond time resolution in gas-phase TEM [3,4], liquid environments present additional challenges that must be addressed to achieve similar progress. The higher density of a liquid medium means the electron beam is much more likely to interact with the liquid itself instead of/in addition to the sample of interest, leading to losses in spatial resolution and often undesirable side reactions. This is exacerbated by imperfections in the liquid cell used to house the sample which can bulge (increasing liquid thickness) or leak evaporation into the microscope (reducing the column vacuum). Material characterization is likewise limited by electron scattering in the medium, which overwhelms the electron energy-loss spectroscopy (EELS) signal at high loss. [5]

Here, we demonstrate the measurement of single-particle plasmon resonances using in-situ liquid-phase scanning TEM EELS despite these complications. We study a model system of individual Au nanoparticles, selected for their strong, anisotropic, low-energy plasmon peaks across a range of wavelengths. We synthesize a variety of particle shapes ranging from $30-100 \mathrm{~nm}$ in size, through both colloidal (reduction of $\mathrm{AuCl}_{4}$ in $\mathrm{CTAB}$ solution) and lithographic (e-beam lithography and metal lift-off) methods onto a specially designed liquid cell chip (Figure 1b). Two such chips are used to enclose a small pocket of liquid for stable imaging and spectroscopy in the TEM (Figure 1a). Most liquid EEL spectra contain an optical gap below its lowest energy absorption ( $~ 8 \mathrm{eV}$ in water) which leaves room for strong plasmon resonances to appear above the background at these low energies. Still, decreasing liquid thickness to preserve zero-loss electrons before they reach our particles helps to magnify these plasmon signals. Thus, we select chip parameters to reduce window bowing and assemble the cell in a crosswindow configuration to minimize liquid thickness at the cell corners.

By selecting particles in the cell located at these corners in thinner liquid regions $(<100 \mathrm{~nm}$ thick $)$, we demonstrate that plasmon resonances can be measured with sub-second total acquisition times while maintaining sufficient signal-to-noise ratios, circumventing the dominant liquid background signal while minimizing interfering electron-induced side reactions. In aqueous media, we observe the expected energy shift relative to vacuum, even with a thin amount of water. Furthermore, we observe that enough spatial and spectral resolution is achieved to differentiate resonances corresponding to spatially dependent visible modes in each shape of particle. For example, across colloidally synthesized Au rods bound to a $\mathrm{SiN}$ membrane, we measure longitudinal and transverse resonances which normally lie at $1.4-1.7 \mathrm{eV}$ and $2.0-2.5 \mathrm{eV}$ respectively in vacuum but experience an approximate $.1 \mathrm{eV}$ redshift in water (Figure 1c). Likewise, for lithographically fabricated 100-nm diameter disks, we map edge modes around $1.8 \mathrm{eV}$ in vacuum, and we observe a similar redshift to around $1.7 \mathrm{eV}$ when immersed in water. These trends 
quantitatively agree with calculated results from BEM methods for the geometries examined. We then measure and compare plasmon resonance energies across distinct chemical environments by changing the media in the liquid cell via flow tubing. Thus, we show that Au particles experience a shift in plasmon resonance arising from the refractive index change of varying liquid media vs. vacuum in agreement with simulations and ex-situ spectroscopy.

Our work demonstrates the ability to accurately measure plasmon energies and their shifts in liquid-phase TEM using EELS. This presents an important technique for in-situ TEM characterization of plasmonic catalysts and a useful tool for monitoring these catalysts during reactions in solution down the road.
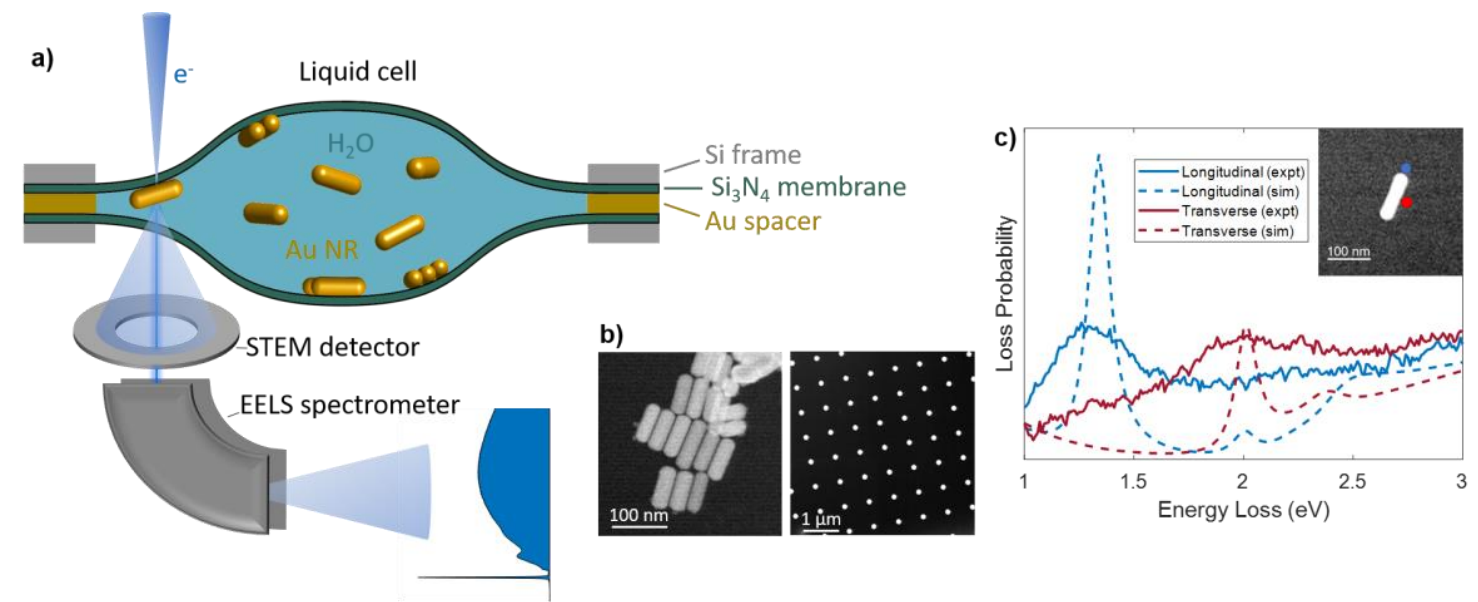

Figure 1. Figure 1. a) Schematic of TEM imaging and EELS acquisition of nanoparticles encapsulated in a liquid cell. b) STEM image of Au nanorods (left) and Au nanodisk array (right) in a 100-nm spacer liquid cell. c) STEM-EELS spectra of overlaid simulated and experimental spatially-resolved plasmon resonances of an Au nanorod in water. The inset shows a STEM-ADF image of the Au nanorod and the electron beam location for measuring the transverse (red) and longitudinal (blue) plasmon energies.

\section{References}

[1] Aslam, U., Rao, V. G., Chavez, S. \& Linic, S. Nat. Catal. 1, 656-665 (2018).

[2] Shin, H. H., Koo, J. J., Lee, K. S. \& Kim, Z. H. Appl. Mater. Today 16, 112-119 (2019).

[3] Vadai, M., Angell, D. K., Hayee, F., Sytwu, K. \& Dionne, J. A. Nat. Commun. 9, 1-8 (2018).

[4] Yang, W. C. D. et al. Nature Materials 18, 614-619 (2019).

[5] Hwang, S., Chen, X., Zhou, G. \& Su, D Adv. Energy Mater. 2, 1902105 (2019). 